\title{
Numerical simulation of 3-D flow around sounding rocket in the lower thermosphere
}

\author{
J. Kurihara ${ }^{1}$, K. -I. Oyama ${ }^{1}$, N. Iwagami ${ }^{2}$, and T. Takahashi ${ }^{3}$ \\ ${ }^{1}$ Institute of Space and Astronautical Science, Japan Aerospace Exploration Agency, Sagamihara, Kanagawa 229-8510, Japan \\ ${ }^{2}$ Dept. of Earth and Planetary Science, Graduate School of Science, University of Tokyo, Bunkyo-ku, Tokyo 113-0033, Japan \\ ${ }^{3}$ Information Science Laboratory, Tokai University, Hiratsuka, Kanagawa 259-1292, Japan
}

Received: 23 July 2005 - Revised: 29 November 2005 - Accepted: 21 December 2005 - Published: 7 March 2006

\begin{abstract}
Numerical simulations using the Direct Simulation Monte Carlo (DSMC) method are known to be useful for analyses of aerodynamic effects on in-situ rocket measurements in the lower thermosphere, but the DSMC analysis of a spin modulation caused by an asymmetric flow around the rocket spin axis has been restricted to the two-dimensional and axially symmetric simulations in actual sounding rocket experiments. This study provides a quantitative analysis of the spin modulation using a three-dimensional (3-D) simulation of the asymmetric flow with the DSMC method. Clear spin modulations in the lower thermospheric $\mathrm{N}_{2}$ density measurement by a rocket-borne instrument are simulated using the rocket attitude and velocity, the simplified payload structure, and the approximated atmospheric conditions. Comparison between the observed and simulated spin modulations show a very good agreement within $5 \%$ at around $100 \mathrm{~km}$. The results of the simulation are used to correct the spin modulations and derive the absolute densities in the background atmosphere.
\end{abstract}

Keywords. Atmospheric composition and structure (Pressure, density and temperature; Thermosphere-composition and chemistry; Instruments and techniques)

\section{Introduction}

Atmospheric in-situ measurements using sounding rockets are affected by aerodynamic effects caused by the supersonic motion of the rocket. For typical rocket experiments in the mesosphere and lower thermosphere region, the aerodynamic condition is generally regarded as a transition regime from continuum flow to free molecular flow. The Direct Simulation Monte Carlo (DSMC) method (Bird, 1994) is widely used to simulate a flow under such a transition regime and applied to a variety of fields. The first application of the DSMC method to rarefied atmospheric flow in a sounding rocket ex-

Correspondence to: J. Kurihara

(kuri@isas.jaxa.jp) periment was made by Bird (1988). Since then, the DSMC method has been recognized as a powerful numerical tool for the analysis of in situ measurements and applied to a wide variety of measurement techniques (e.g. Gumbel et al., 1998; Horányi et al., 1999; Gumbel, 2001a,b; Rapp et al., 2001; Sternovsky et al., 2004).

An angle of attack, $\alpha$, which is defined as the angle between the rocket spin axis and the velocity vector, is an important parameter for the flow pattern around the rocket. If $\alpha$ has a value other than $0^{\circ}$, the flow pattern becomes asymmetric with respect to the rocket spin axis. Previous studies using the DSMC method for actual sounding rocket experiments have been restricted to the two-dimensional (2-D) and axially symmetric simulations, and did not treat the angles of attack other than $0^{\circ}$ directly. Rapp et al. (2001) utilized the result of the DSMC simulation (Gumbel, 2001b) indirectly, together with a conversion factor experimentally determined by wind tunnel measurements as a function of $\alpha$, and corrected the aerodynamical effect in the asymmetric flow. However, a fully 3-D simulation of the asymmetric flow is required for the analysis of a spin modulation, which is the variation of the measured quantity synchronized with the rocket spin frequency. The spin modulation is usually observed with side-looking instruments when $\alpha \neq 0^{\circ}$ (Gumbel, 2001a).

This paper reports on a quantitative analysis of the spin modulation using the 3-D simulation of the flow around the sounding rocket. Clear spin modulations were seen in the atmospheric $\mathrm{N}_{2}$ density measurement in the lower thermosphere with a rocket-borne $\mathrm{N}_{2}$ temperature instrument (NTV), which is a side-looking instrument using the Electron Beam Fluorescence (EBF) technique (Kurihara and Oyama, 2005). The angle of attack monitored by an attitude sensor onboard the rocket is used as an input parameter in the numerical simulation. The DSMC method is used to simulate the asymmetric density fields around the rocket. The results of the simulation are compared with the measured density variations and applied to correct the spin modulations and determine the absolute densities. 


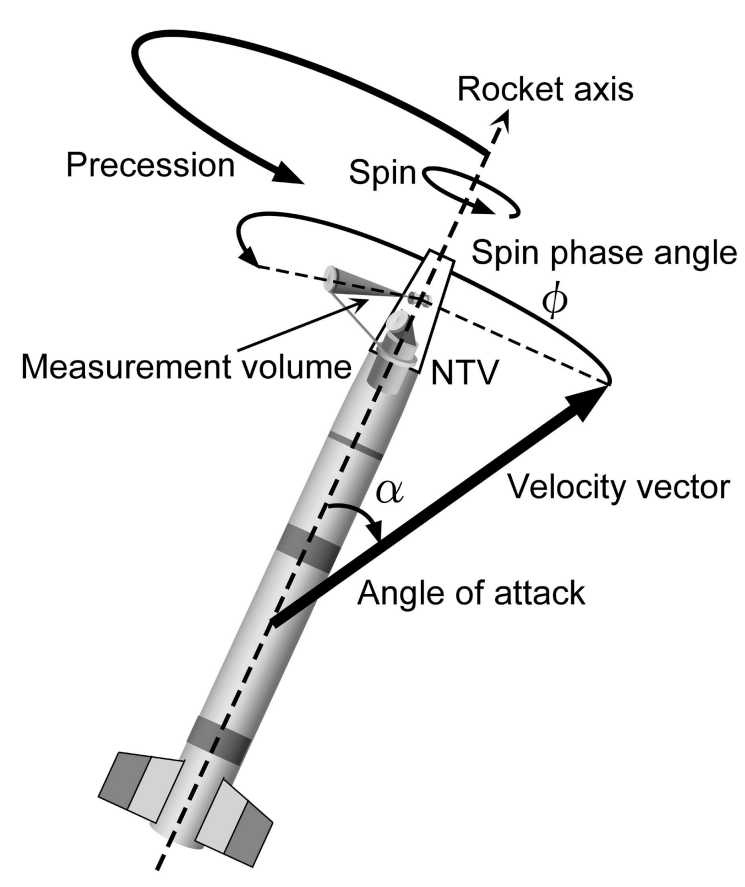

Fig. 1. Definition of the rocket attitude and motion.

\section{Rocket observation}

The atmospheric $\mathrm{N}_{2}$ density measurement was conducted in Japan on 6 February 2002 (Kurihara et al., 2003). The sounding rocket on which the NTV instrument was installed was launched at 19:30 LT from Kagoshima Space Center and reached an apogee altitude of $151.8 \mathrm{~km}$ at $190 \mathrm{~s}$ after the launch. The measurements by NTV were made in the 100.0$151.8 \mathrm{~km}$ altitude region during the ascent of the flight and in $151.2-95.0 \mathrm{~km}$ during the descent. The NTV instrument consists of an electron gun to excite and ionize the ambient $\mathrm{N}_{2}$ and a sensitive spectrometer to detect the subsequent fluorescence spectrum of the $\mathrm{N}_{2}^{+}$1st Negative $(1 \mathrm{~N})$ bands based on the EBF technique. This instrument is designed to measure vibrational and rotational temperatures, in addition to the number density of $\mathrm{N}_{2}$. The temperatures are derived from the band shape of the $\mathrm{N}_{2}^{+} 1 \mathrm{~N}$ bands, and the number density is determined from the intensity of the band. Further details of the instrument and its calibration in the laboratory are given by Kurihara and Oyama (2005). The exposure time for each spectrum is $240 \mathrm{~ms}$ and the average spin period of the rocket is about $920 \mathrm{~ms}$; roughly four spectra are obtained per spin. The measurement volume of NTV is located to the side of the payload, and thus NTV scans the density field around the rocket with the spin motion. As a result, the number density derived from one spectrum is the average value of the density field scanned over one-fourth of the spin period. The measured densities are strongly contaminated by aerodynamical effects and the spin modulations are clearly observed in the density profiles. In this paper, the density data measured only in the ascent are studied because the angle of
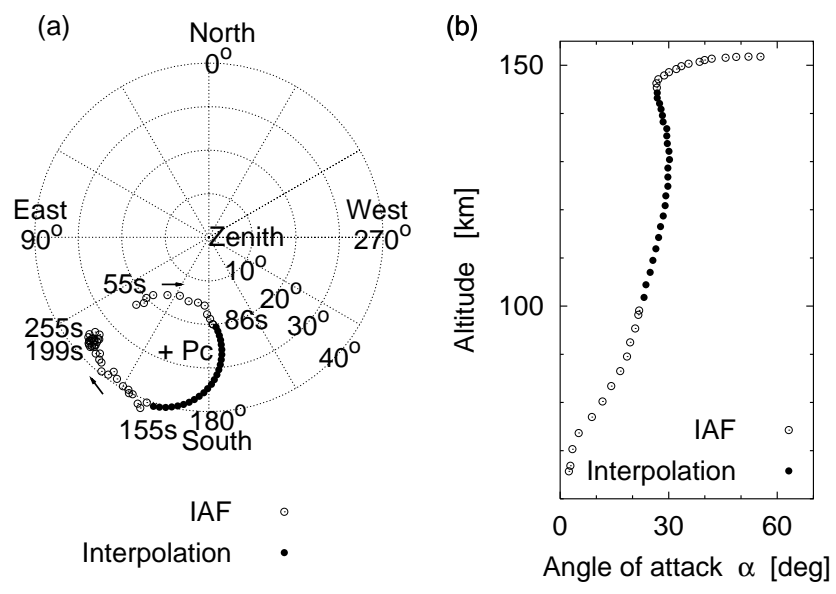

Fig. 2. (a) Direction of the rocket axis. The IAF measurements (open circles) were obtained only 57-86 and 155-255 s after the launch, and the missing data during $86-155 \mathrm{~s}$ are interpolated (closed circles) by assuming a stable precession, whose center is indicated by "Pc". Arrows indicate the direction of the precession. (b) Angle of attack variation in the ascent derived from the attitude data in Fig. 2a.

attack measurement by the attitude sensor is not available in the descent as described below.

Figure 1 shows the definition of the rocket attitude and motion, and the attitude generally changes with a periodic motion, called precession, which is a rotation of the rocket axis, whereas spin is a rotation around the rocket axis. In this experiment, the rocket attitude and motion are measured with the Geomagnetic Aspectmeter (GA) and the Imaging Attitude Finder (IAF) on board the rocket. GA has two sensors to measure the horizontal and vertical components of the geomagnetic field vector with respect to a cross sectional plane of the rocket and can obtain the spin period of the rocket and the angle between the rocket axis and the geomagnetic field vector. IAF is a two-dimensional star sensor using a multi-anode photomultiplier tube and can determine the direction of the rocket axis with an accuracy of 2 degrees. However, the fluorescence induced by NTV was much brighter than expected, and the output signal of IAF was saturated most of the time during the emission of the electron beam. Figure 2 a shows the direction of the rocket axis in the alt-azimuth coordinates. The IAF measurements are limited to $57-86 \mathrm{~s}(66-99 \mathrm{~km}$ altitudes in the ascent) and to $155-$ $255 \mathrm{~s}$ (from $145 \mathrm{~km}$ in the ascent to $134 \mathrm{~km}$ in the descent) after the launch. The rocket was separated after the apogee (199s) into mother/daughter payloads. The mother payload includes a solid rocket motor and a Langmuir probe, and the daughter payload includes NTV and the attitude sensors. The purpose of the separation is to electrically isolate the Langmuir probe from NTV, because the electron beam emission by NTV causes significant charging of the rocket body for the Langmuir probe measurement of the ambient plasma. After the rocket separation, the precession of the rocket became smaller and more rapid, and the direction of the rocket axis 
was almost stationary. The missing attitude data during 86$155 \mathrm{~s}$ are interpolated by assuming a stable precession of the rocket axis before the separation. The center and the radius of the precession circle are determined by a least-squares fitting to the IAF data points. The interpolated attitude data are consistent with the variation of the geomagnetic field vector measured by GA. Figure $2 b$ shows the angles of attack derived from the above attitude data in the ascent. The angle of attack varies between $20-60^{\circ}$ during the NTV measurement and is large enough to cause the asymmetric flow.

In order to analyze the characteristics of the spin modulation, the spin phase angle distribution of relative density changes is examined. The spin phase angle, $\phi$, is defined as an angle between the rocket velocity vector and the look direction of NTV in a plane perpendicular to the rocket axis, as shown in Fig. 1. Accordingly, the location of the measurement volume with respect to the flow direction is represented as a function of $\phi ; \phi=0^{\circ}$ corresponds to the front side of the rocket and $\phi=180^{\circ}$ corresponds to the rear side of the rocket. The relative density change is defined by the ratio of the measured density to the smoothed density. Considering that four density data are sampled per spin, it is appropriate to perform a running average over five data points for smoothing the density profile. Figure 3 a shows the measured and smoothed density profile between $100-105 \mathrm{~km}$ altitudes during the ascent of the rocket. Figure $3 \mathrm{~b}$ shows the relative density changes plotted as a function of $\phi$. The relative density change shows a $10 \%$ increase at $\phi=0^{\circ}$ and a $10 \%$ decrease at $\phi=180^{\circ}$. These features are typical spin modulation, commonly called "ram/wake modulation", originating from compression/rarefaction in the density field around the rocket. Similar characteristics of the relative density change are found in the other altitude ranges, as shown later.

\section{Direct simulation Monte Carlo calculations (DSMC)}

The DSMC method is applied to a fully 3-D simulation of the flow around the rocket. Details of the DSMC method are described in Bird (1994). The most fundamental principle of the DSMC method is an approximation that the motions and collisions of molecules are uncoupled during a short time step. For the realization of this approximation, the time step should be sufficiently shorter than the mean free time of the collisions. The approximation also requires the size of sampling cells to be larger than the moving distances of molecules during the time step, but to be small compared to the length scale of local flow gradients, as well as the mean free path. The length scale of local gradients depends on the size and shape of the object in the flow. This means that the size of the cells and the time step have upper limits. These two requirements basically determine the total time steps and the total number of the cells, namely computing capacity required for the simulations. In rarefied atmospheric flow simulations, the mean free time and the mean free path increase rapidly with an increase in altitude. If the time step reaches at the upper limit, the total time steps of the simulation grow (a)

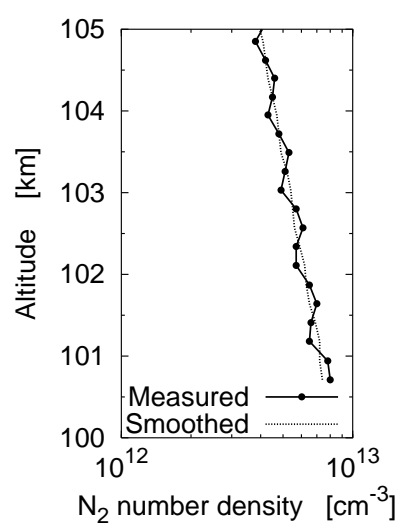

(b)

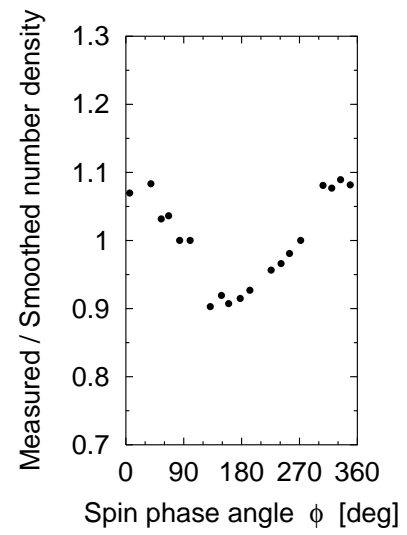

Fig. 3. Effect of spin modulation on the measured density profile between $100-105 \mathrm{~km}$ altitudes in the ascent. (a) Measured and smoothed profiles. (b) Relative density changes plotted as a function of the spin phase angle.

with increasing altitude because the sampling of macroscopic properties in the flow is performed at a time interval comparable to the mean free time. On the other hand, the total number of the cells increases with decreasing altitude, because the size of the cell should be smaller than the mean free path. As a result, the DSMC simulation for the flow around a sounding rocket is generally efficient at the altitudes of 100 $120 \mathrm{~km}$, and more powerful computing capacity is needed at lower and higher altitudes. For this reason, the fully 3D simulation for the lower thermospheric conditions in this study are practical for present-day PCs but it is still difficult to accomplish the calculations for the mesospheric conditions. Details of the numerical code and cell schemes used in this simulation have been provided by Kurihara (2004).

The numerical simulation is performed by providing the necessary input parameters, viz., angle of attack, rocket velocity, geometry of the payload, and background atmospheric conditions. As mentioned previously, the angle of attack is a critical parameter controlling the asymmetry of the flow. The rocket velocity and the background atmospheric temperature are also important for determining the Mach number $(M a)$ of the flow. The tangential velocity components caused by the spin of the rocket are not considered in the simulations because they can be neglected compared to the supersonic speeds of the rocket. For example, the tangential velocity of the spin is about $1 \mathrm{~m} / \mathrm{s}$ at the surface of the rocket, while the rocket velocity is more than $1 \mathrm{~km} / \mathrm{s}$. Although the actual payload has a complex geometry, the simulated payload is simplified as a truncated cone. However, the complex geometry of the payload is of minor importance for the simulation, because the measurement volume of NTV is much larger than the size of the components of the payload. The background atmospheric parameters, such as atmospheric temperature and density, should be carefully considered in this simulation, because the measured temperature and density profiles are significantly different from the 


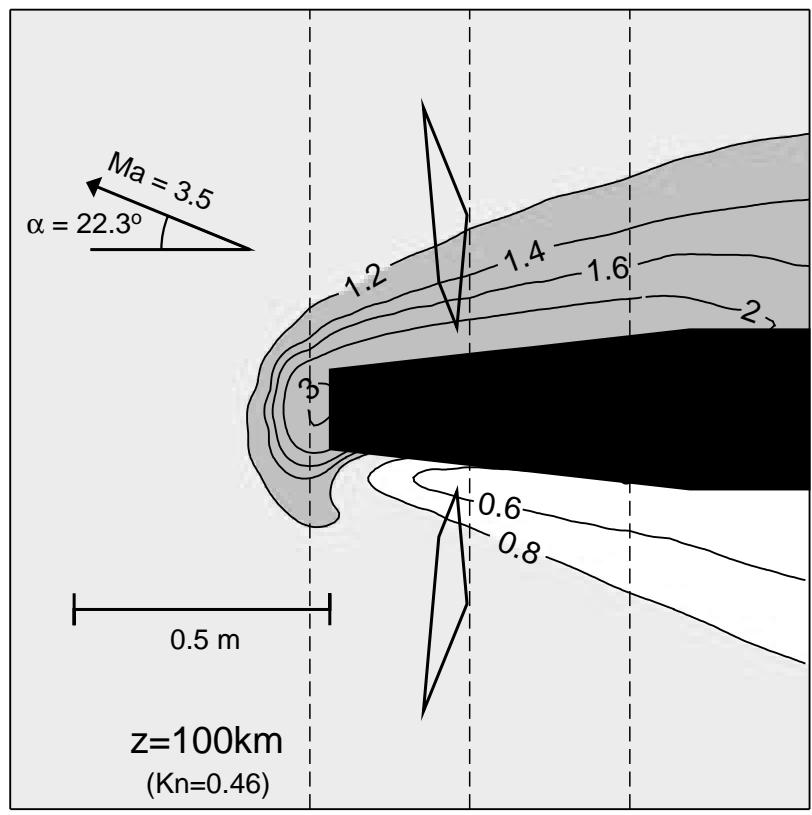

(a) (a)

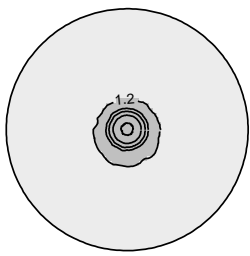

(b)

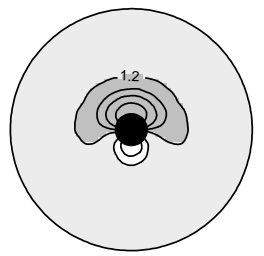

(c)

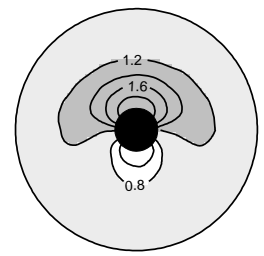

Fig. 5. Cross-sectional views of the calculated relative density field at (a), (b), and (c) in Fig. 4.
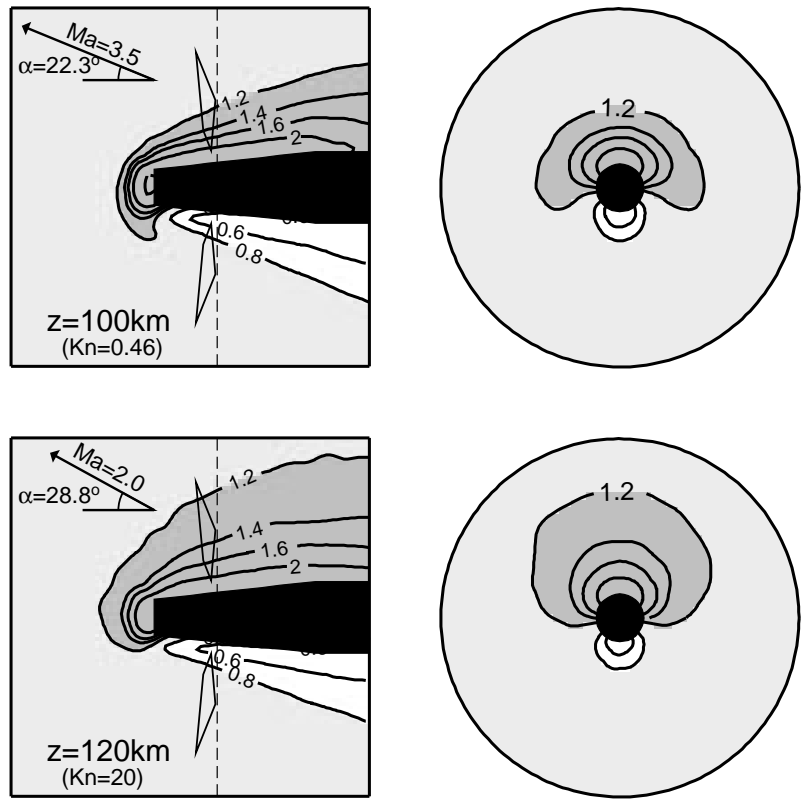

and then converted to the total number densities using the relative composition from the MSISE-90 atmospheric model (Hedin, 1991). The present study uses a hard sphere model for simulating particles in the flow and simplifies the atmospheric molecules to a single gas (air). Assuming that the particle is a hard sphere with a diameter of $d$, the mean free path of the gas particles, $\lambda$, is expressed as a function of the total number density of the particles, $n$, by $\lambda=1 /\left(\sqrt{2} \pi d^{2} n\right)$. The rarefied gas flow is characterized by the Knudsen number, $K n=\lambda / L$, where $L$ is the characteristic length of the object. In the case of the flow around the sounding rocket, a diameter of the rocket is usually taken as the characteristic length.

Results of the above-described DSMC calculations provide the complex 3-D nature of the flow. As an example, Fig 4 shows a calculated relative density field, which is the ratio of the calculated density to the background atmospheric density. This figure is presented in a cross-sectional plane, including the rocket spin axis and the velocity vector, and the simulation is done for the flight condition corresponding to the altitude of $100 \mathrm{~km}$. The black region represents the payload geometry, and the quadrangular regions indicate the measurement volume of NTV. The arrow indicates the velocity vector of the rocket, and in turn, the supersonic flow moves in the opposite direction to the arrow. The flow properties $\alpha, M a$, and $K n$, are $22.3^{\circ}, 3.5$, and 0.46 , respectively.
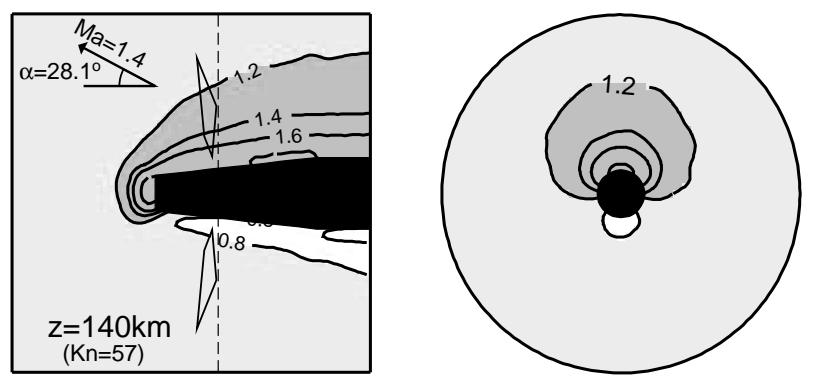

Fig. 6. Calculated relative density fields for 100,120 , and 140-km altitudes. Right panels show the cross-sectional views of the relative density fields at the dashed lines in the left panels.

The relative density field is shown by the contours and gray scale. The density is doubled near the front surface of the payload by compression and decreases $50 \%$ right behind the payload by rarefaction. The measurement volume is located at the higher density region in the front side of the payload and at the slightly lower region in the rear side. This situation illustrates the ram/wake modulation seen in the observation. Figures $5 \mathrm{a}, 5 \mathrm{~b}$, and $5 \mathrm{c}$ show cross-sectional views 

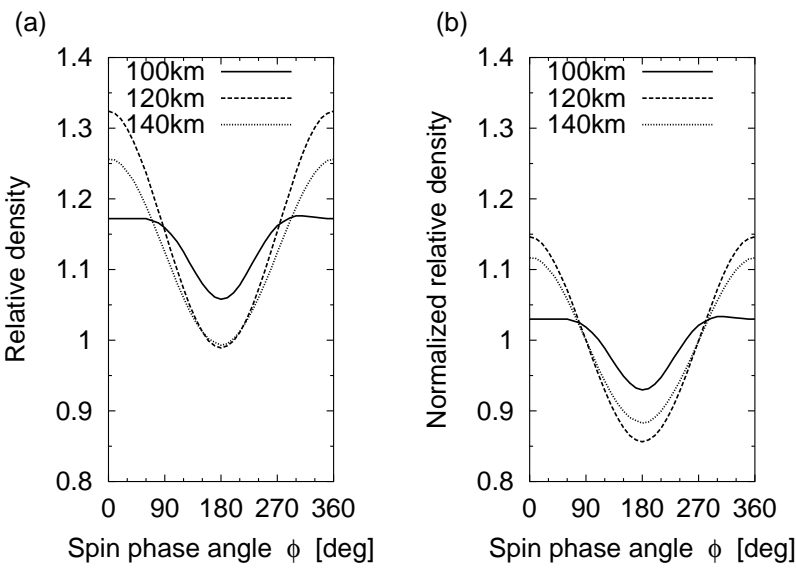

Fig. 7. (a) Relative densities simulating the NTV measurement and (b) normalized relative densities over the spin.

of the relative density field perpendicular to the rocket axis at (a), (b), and (c) in Fig. 4, respectively. The axially asymmetric nature of the flow is clearly seen in Figs. 5b and 5c. In particular, the folding density field around the payload is the most remarkable feature of the axially asymmetric flow, which can be reproduced only by the 3-D simulation. Similar features were found in the wind tunnel experiments but not in the 2-D simulations (Gumbel, 2001a), so far.

Results of the calculations for various altitudes are shown in Fig. 6. The arrows in the left panels are the same as for Fig. 4, but the relative lengths of the arrows correspond to the rocket velocities at the respective altitudes. In the left panels of Fig. 6, the relative density gradients around the rocket at $100 \mathrm{~km}$ are much steeper than at $120 \mathrm{~km}$, and the relative density field undergoes a drastic change from a discrete pattern to a diffuse one between $100-120-\mathrm{km}$ altitudes, but there is little change in the pattern above $120 \mathrm{~km}$. These features of the flow patterns are more noticeable in the right panels of Fig. 6, and the folding density field at $100 \mathrm{~km}$ is substantially reduced above $120 \mathrm{~km}$. These changes are mainly attributed to the transition from continuum flow $(K n<0.1)$ to free molecular flow $(K n \gg 10)$, and the drastic change in the relative density field corresponds to a change in the property of the flow at around $110 \mathrm{~km}$ altitude, where $K n \sim 1$ : the mean free path of the gas particles is comparable to the characteristic length of the object. Although the Mach number also changes with altitude, a decrease in the Mach number decreases the magnitude of the maximum compression and rarefaction in the free molecular flow regime above $120 \mathrm{~km}$. Gumbel (2001a) reported the altitude development with the 2-D simulation for $\alpha=0^{\circ}$, which shows that the shock effects decrease and focus towards the ends of the payload at high altitude. In the present 3-D simulation, the angles of attack are sufficiently large to form a shock front all along the payload and maintain a strong ram/wake modulation, even at high altitude.

The spin modulations are reproduced from these simulations for comparison with the observations. The exact value

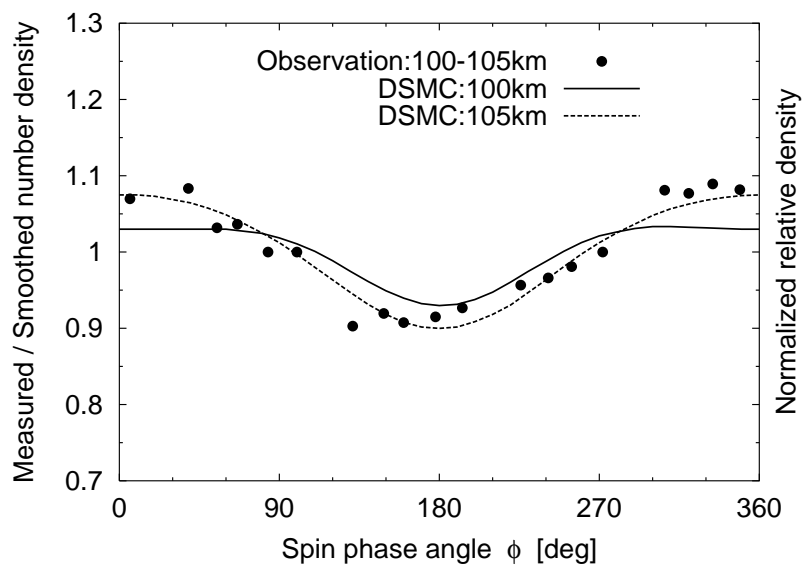

Fig. 8. Relative density changes for the observation between the $100-105-\mathrm{km}$ altitudes and the DSMC results for 100 and $105 \mathrm{~km}$. The vertical axis corresponds to the measured/smoothed value for the observation and the normalized relative density for the DSMC results.

of the measured density cannot be reproduced straightforwardly from this simulation, because the background atmospheric density used in the simulation is based on the contaminated $\mathrm{N}_{2}$ density from the observation and the relative composition from the empirical model. However, the results of the above simulations for the various altitudes show that the changes in the relative density field are small compared with those of the background density. This suggests that a simulated relative density can closely approximate the measured relative density, even if the exact value of the background density is unknown. Therefore, the spin modulation is simulated by the following two steps: the relative densities are simulated according to the NTV measurement as a function of $\phi$, and then normalized over the spin, by dividing the relative densities by the spin-average value of the relative densities. This normalized relative density is defined as a simulated spin modulation. Figure 7 a shows the relative densities simulated for various altitudes, and Fig. $7 \mathrm{~b}$ shows the normalized relative densities. It should be noted that the relative densities in Fig. 7a are greater than one at any spin phase angle and any altitude. Even at $\phi=180^{\circ}$, the density is higher than or equal to the background atmospheric density, because the region which the measurement volume of NTV scans is dominated by the higher densities, as shown in the cross-sectional views in Fig. 6, and the density is derived from the scan over a wide range of angles $\left(\sim 90^{\circ}\right)$. On the other hand, the normalized relative densities are greater than one at the front side but less than one at the rear side, as the result of the normalization. The results of these simulations indicate that the background atmospheric quantity is not always obtained by a running average smoothing of the spin modulation observed with side-looking instruments. 

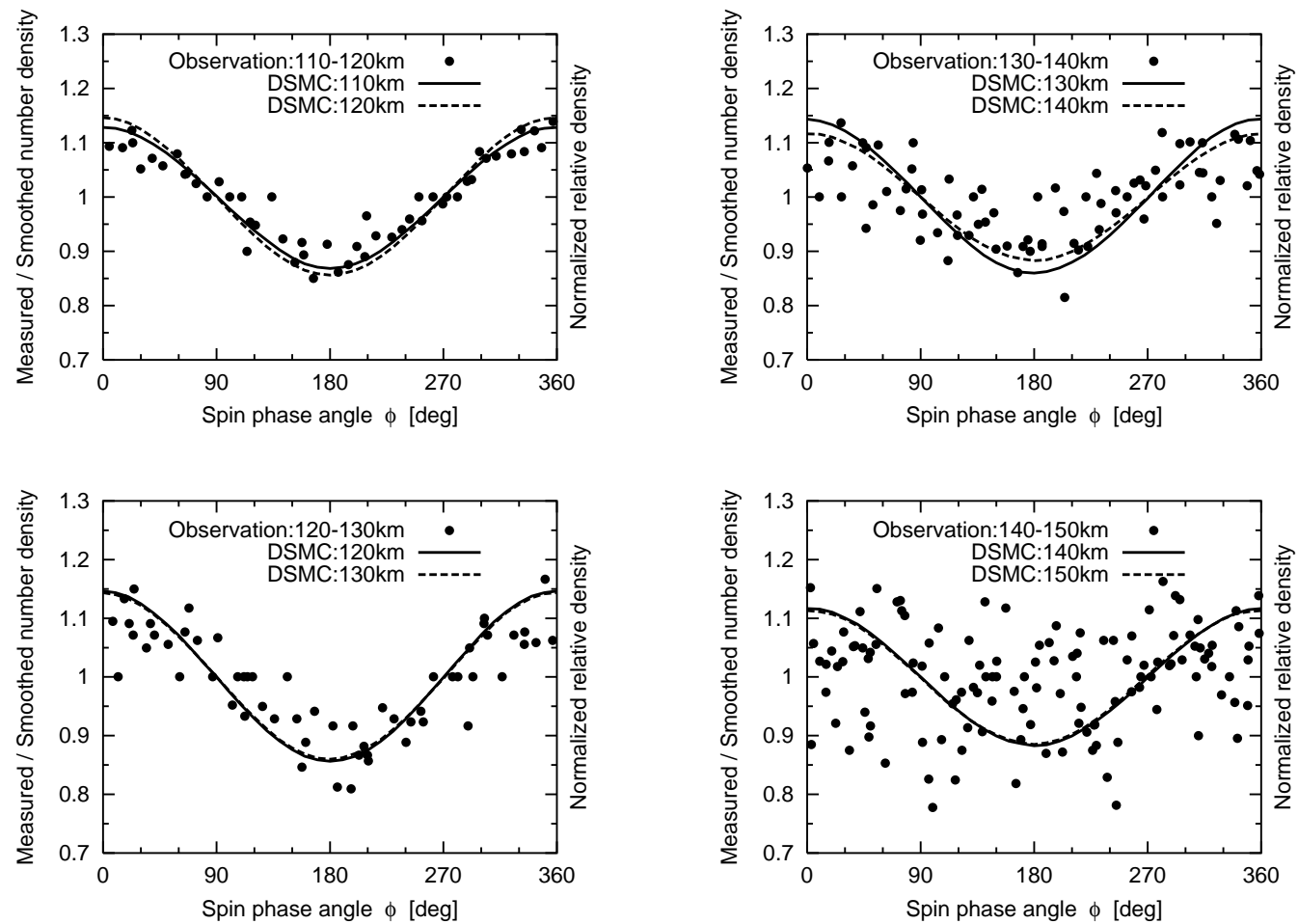

Fig. 9. Relative density changes for the observation and the DSMC results for various altitude regions.

\section{Correction of the aerodynamic effects}

The spin modulations from the observations and the DSMC results between the 100-105-km altitudes are compared, as shown in Fig. 8. In this altitude region, the observations and the DSMC results are in fairly good agreement within $5 \%$. The precision of the density measurement is less than $1 \%$ at $100 \mathrm{~km}$ and much smaller than the difference between the observation and the simulation. The difference comes from both the uncertainty of the simulation model and smallscale perturbations in the background atmosphere. Despite the simplifications and approximations in the model, the simulation is remarkably accurate in this altitude region. Similar comparisons at other altitudes are shown in Fig. 9. The random variations in the observed data grow exponentially with altitude, because the uncertainty of the density measurement is inversely proportional to the density. The relative density changes for the observations in Fig. 9 become scattered at higher altitudes, mainly because of this growth in the measurement uncertainty and, consequently, the spin modulation from the observations loses its character. It is therefore difficult to quantify the accuracy of the simulations above 130$\mathrm{km}$ altitude, but there is a clear deviation between the simulations and observations. On average, amplitudes of the spin modulation in simulations are larger than that in the observations, while the basic features of ram/wake modulation are reasonably reproduced at all altitudes. These results imply that some simplifications and approximations in the model become less valid above $130 \mathrm{~km}$. The simplification of a sin- gle gas used in this model may be critical for simulations at high altitude because aerodynamic effects depend on the composition that changes rapidly with altitude in the lower thermosphere.

Since the validity of the present simulation is confirmed by the agreement between the observed and simulated spin modulations below $130-\mathrm{km}$ altitude, the simulated relative densities are applied to correct the measured densities and determine the absolute densities. The correction is done by dividing the measured density by the relative density from the simulation. Figure 10 shows altitude profiles of the measured and corrected densities. Note that the corrected profile above $110 \mathrm{~km}$ is smoothed by a running average, to cancel the uncertainty, because the large random variations remain in the measured densities at higher altitudes. However, even below $110 \mathrm{~km}$, a smooth density profile is successfully obtained, and the spin modulation is significantly reduced. This result also supports the validity of the simulation. In addition, the corrected density profile is lower than the measured density profile at almost all altitudes. As mentioned above, the reason is that the measured density is derived from a wide range of the scan, and the scanning region is dominated by the high relative densities.

Correction of the aerodynamic effects on in-situ measurements and the determination of the absolute density in the lower thermosphere are of increasing importance because the existing reference models turned out to be inaccurate in some cases. Rapp et al. (2001) have determined a total of $20 \mathrm{ab}-$ solute density profiles from rocket-borne measurements with 


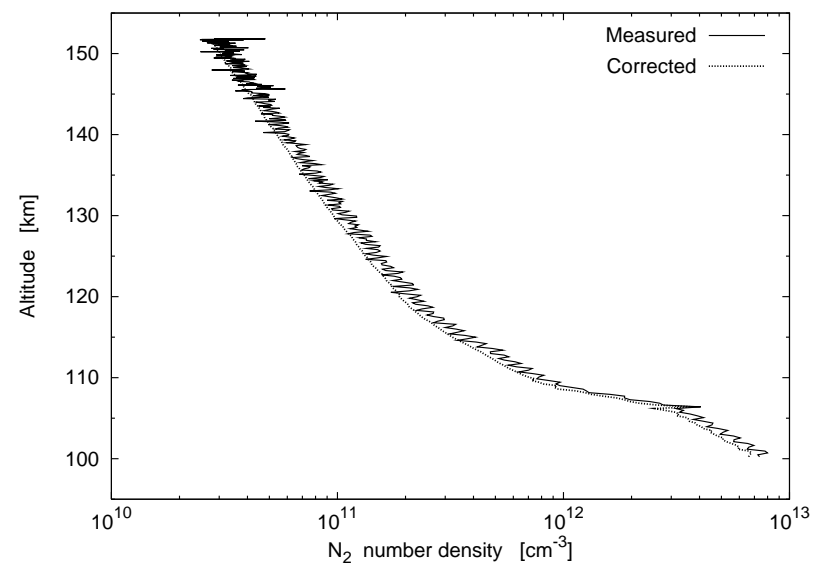

Fig. 10. Altitude profiles of the measured and corrected density.

ionization gauges at high latitudes and found large discrepancies between their results and the reference atmospheres above $85 \mathrm{~km}$ altitude. In the present study, the derived absolute $\mathrm{N}_{2}$ density profile has a steep gradient at an altitude range between $106-110 \mathrm{~km}$, and at around $115-120 \mathrm{~km}$, the $\mathrm{N}_{2}$ density is significantly different from that of the empirical atmospheric model. Details of this structure will be discussed in a future paper. The results of the simulations in this study demonstrated that the flow pattern around the rocket drastically changes between $100-120-\mathrm{km}$ altitudes due to the transition from continuum flow to free molecular flow, but it is evident from Fig. 10 that the density drop at $106 \mathrm{~km}$ is not caused by the aerodynamical effects.

\section{Conclusions}

The results of the 3-D numerical simulation for the asymmetric flow around the sounding rocket using the DSMC method are compared with the spin modulations in the atmospheric $\mathrm{N}_{2}$ density measurement by the rocket-borne instrument. The observed and simulated spin modulations show generally a good agreement, even though the payload geometry and the background atmosphere are simplified in the simulation model. The simulated spin modulations with larger amplitudes than the observed at high altitude imply that compositional change may be important for the rarefied flow simulations in the lower thermosphere. The absolute $\mathrm{N}_{2}$ density profile is obtained by the correction of the spin modulations.

The present study provides quantitative analysis to derive the absolute density, as well as qualitative illustration of the spin modulation. The 3-D simulation model used here is applicable to other side-looking instruments, such as a resonance fluorescence instrument for atomic oxygen measurement, which usually suffers a crucial contamination by the spin modulation. In addition, if the 3-D simulations are accurate enough to identify which spin angle contains the background values (or close to them), this information can be used as a filter on the measurements instead of smoothing all the data, which, by definition, contains some bad data points deviated from the background values.

Acknowledgements. The authors would like to acknowledge T. Abe (ISAS/JAXA) for discussion on the aerodynamic effects and introduction to the DSMC method. The authors also thank the rocket launching group of ISAS and all related government institutions for conducting the successful experiments.

Topical Editor U.-P. Hoppe thanks two referees for their help in evaluating this paper.

\section{References}

Bird, G. A.: Aerodynamic effects on atmospheric composition measurements from rocket vehicles in the thermosphere, Planet. Space Sci., 36, 921-926, 1988.

Bird, G. A.: Molecular gas dynamics and the direct simulation of gas flows, Oxford Univ. Press, Oxford, 1994.

Gumbel, J.: Aerodynamic influences on atmospheric in situ measurements from sounding rockets, J. Geophys. Res., 106, 10553 10563, 2001a.

Gumbel, J.: Rarefied gas flows through meshes and implications for atmospheric measurements, Ann. Geophys., 19, 563-569, 2001b,

SRef-ID: 1432-0576/ag/2001-19-563.

Gumbel, J., Murtagh, D. P., Espy, P. J., and Witt, G.: Odd oxygen measurements during the Noctilucent Cloud 93 rocket campaign, J. Geophys. Res., 103, 23 399-23 414, 1998.

Hedin, A. E.: Extension of the MSIS thermosphere model into the middle and lower atmosphere, J. Geophys. Res., 96, 1159-1172, 1991.

Horányi, M., Gumbel, J., Witt, G., and Robertson, S.: Simulation of rocket-borne particle measurements in the mesosphere, Geophys. Res. Lett., 26, 1537-1540, 1999.

Kurihara, J.: Energetics and structure of the lower thermosphere observed by sounding rocket experiment, $\mathrm{Ph}$. D. thesis, The University of Tokyo, 2004.

Kurihara, J. and Oyama, K.-I.: Rocket-borne instrument for measuring vibrational-rotational temperature and density in the lower thermosphere, Rev. Sci. Instrum., 76, 083101, doi:10.1063/1.1988189, 2005.

Kurihara, J., Oyama, K.-I., Suzuki, K., and Iwagami, N.: Vibrational-rotational temperature measurement of $\mathrm{N}_{2}$ in the lower thermosphere by the rocket experiment, Adv. Space Res., 32, 725-729, 2003.

Rapp, M., Gumbel, J., and Lübken, F.-J.: Absolute density measurements in the middle atmosphere, Ann. Geophys., 19, 571580, 2001,

SRef-ID: 1432-0576/ag/2001-19-571.

Sternovsky, Z., Holzworth, R. H., Horányi, M., and Robertson, S.: Potential distribution around sounding rockets in mesospheric layers with charged aerosol particles, Geophys. Res. Lett., 31, L22101, doi:10.1029/2004GL020949, 2004. 